\title{
Cerebral infarction in childhood bacterial meningitis
}

\author{
R D S Y DER, J STOVRING, A H CUSHING, L E D A IS, A N D \\ T L H A R D Y \\ From the Departments of Neurology, Radiology, and Pediatrics, University of New Mexico \\ Medical Center, Albuquerque, New Mexico
}

SUMMARY Forty-nine children with complicated bacterial meningitis were studied. Thirteen had abnormalities on computed tomography compatible with the diagnosis of brain infarction; one had a brain biopsy with the histological appearance of infarction. Factors exist in childhood bacterial meningitis which are associated with the development of brain infarction.

Although antibiotics have been successful in eradicating the infecting organism in many cases of childhood bacterial meningitis, major neurological complications continue to occur. ${ }^{1-3}$ For many decades physicians have relied upon necropsy to determine the pathogenesis of these complications. ${ }^{4-6}$ Only limited help has been obtained from cerebral arteriography and pneumoencephalography. ${ }^{7-8}$ The complexity and risk of such procedures prevent wide use. ${ }^{9-10}$ Recent application of computed tomography of the brain (CT) has provided a unique and relatively simple noninvasive radiographic method for examining those children with bacterial meningitis who developed neurological complications. ${ }^{11-14} \mathrm{CT}$ has provided additional information concerning the pathogenesis of the neurologic complications.

This report describes a four-year experience with $\mathrm{CT}$ in acute childhood bacterial meningitis and discusses clinical correlations with the CT finding of infarction.

\section{Methods}

A retrospective study of bacterial meningitis in childhood was undertaken at the University of New Mexico Medical Center for the years 1975 to 1979. The University of New Mexico Medical Center provides primary care and also serves as a referral centre for the region. CT was performed on all children with bacterial meningitis whose illness was complicated by seizures, hemiparesis, persistent fever, persistent full fontanelle, or prolonged alteration in mental

Address for reprint requests: Dr RD Snyder, Department of Neurology, University of New Mexico Medical Center, Albuquerque, New Mexico, 87131, USA.

Accepted 5 May 1981 status. Ninety-eight consecutive children with the diagnosis of bacterial meningitis were reviewed retrospectively. Forty-nine children had CT because of the above complications, their ages ranging from three weeks to five years with the majority being under one year of age. Five patients also had followup CT performed six months to two years after the acute illness.

The diagnosis of infarction was based on CT evidence of single or multiple circumscribed or wedgeshaped areas of decreased density without major mass effect. In two patients these areas showed contrast enhancement. When repeat CT was performed one or more months later, the areas of infarction became cystic in appearance (fig 1). Initial CT in the patients with infarction was performed between three and 100 days after the onset of symptoms of meningitis. Seven patients had their initial CT within nine days of onset. The ages of the patients with infarction ranged from three weeks to 11 months. Twelve were males.

An attempt was made to correlate the presence of infarction with seizures, hemiparesis, ventricular enlargement, cerebrospinal fluid findings, bacteriology, antibiotic treatment, brain pathology, and outcome. An EMI Mark I Scanner with a $160 \times 160$ matrix was used in this study.

\section{Results}

\section{Infarction}

Twenty-seven per cent (13 of 49) of the children who had CT demonstrated one or more abnormalities compatible with the diagnosis of infarction. In nine patients the areas of infarction were predominantly unilateral.

In one child with CT findings of infarction (fig 2) a brain biopsy from a low density area 

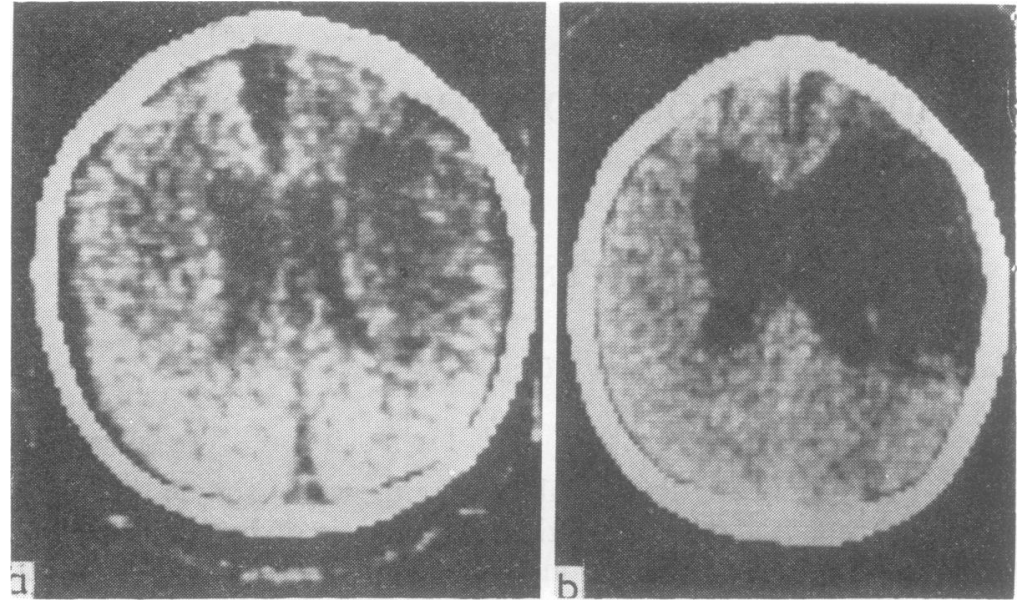

Fig 1 (a) CT performed during acute $H$ influenzae meningitis showing right frontal low density area. Age 6 months. (b) Repeat CT three years later showing cystic appearance suggesting old infarction with ventricular enlargement.

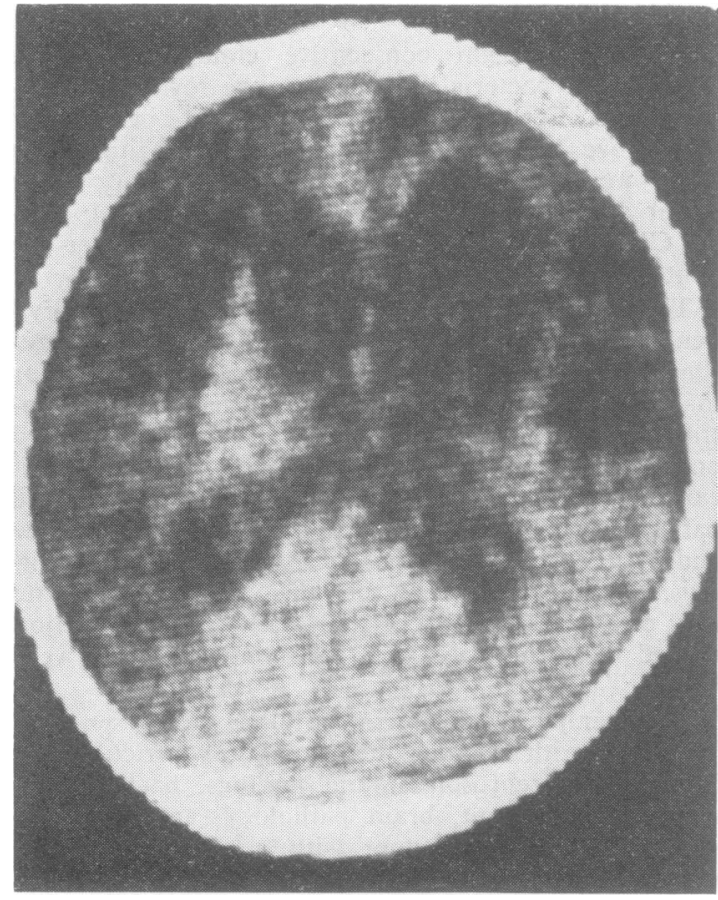

Fig $2 C T$ performed 2 months after $H$ influenzae meningitis. Age 5 months. Multiple circumscribed frontal low density areas. Ventricular enlargement.

was performed two months after the acute illness. The biopsy demonstrated a histologic appearance compatible with the diagnosis of infarction. The cortex showed heavy gliosis. Lipid-laden macroprages were present. Reactive astrocytes and macrophages were found in the deep cortex and superficial white matter (fig 3 ).

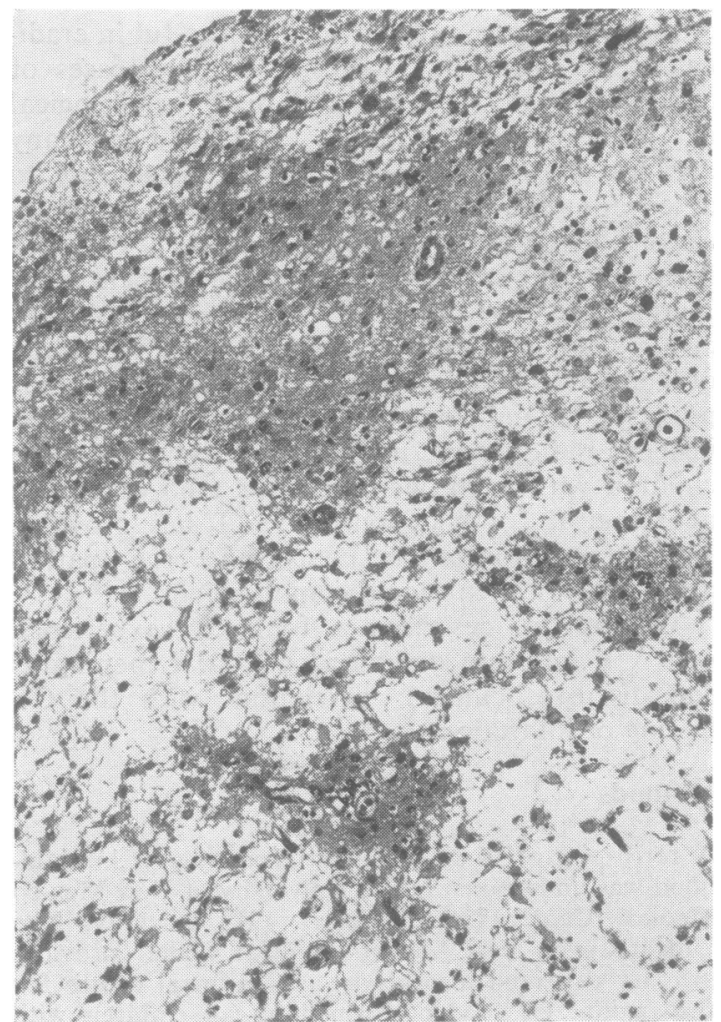

Fig 3 Left frontal cortex biopsy. The upper layers of the cortex show heavy gliosis and contain scattered lipid-laden macrophages. The deep layers contain a loose network of eosinophilic fibres, lipid laden macrophages, reactive astrocytes, and numerous small blood vessels. No neurons are seen (haematoxylin and eosin, original magnification $\times 80$ ). 


\section{Seizures}

The overall incidence of seizures in our entire series of children with bacterial meningitis was $51 \%$. All of the patients with infarction had seizures; nine had focal seizures. In the nine patients with CT evidence of predominantly unilateral infarction, five had seizures which were predominantly contralateral to the area of infarction.

\section{Hemiparesis}

Five of the nine patients with infarcts which were predominantly unilateral had contralateral hemiparesis. The remainder had tetraparesis, perhaps reflecting the bilateral nature of the cerebral lesions even in cases where the infarcts were predominantly unilateral on $\mathrm{CT}$.

\section{Ventricular enlargement}

CT evidence of ventricular enlargement occurred in 11 of the patients with CT evidence of infarction (figs 1 and 2). Two of these also had a full fontanelle. Two of the patients with normal fontanelle pressure had continued ventricular enlargement during external ventricular drainage (fig 4). Follow-up scans, performed in five of these children, showed persistent enlargement without acceleration in the rate of increase of head circumference and an actual decrease in the rate in three patients.

\section{Laboratory findings}

Initial cerebrospinal fluid white cell count in patients with CT evidence of infarction was over 1000 cells in seven cases and was unknown in two. Initial cerebrospinal fluid glucose was $0.6 \mathrm{mmol} / 1$ or less in seven patients and was unknown in three.

The bacteria cultured from the children with cerebral infarction were $H$. influenzae in eight, $S$. pneumoniae in three, and $S$. pyogenes (beta haemolytic) in two.

\section{Antibiotic treatment}

Of the 13 patients with CT evidence of infarction, appropriate antibiotic therapy was begun within two days of the onset of symptoms suggesting meningitis in nine. The longest delay in starting appropriate antibiotic therapy was six days, in two patients. Inappropriate antibiotic therapy or inappropriate dose of antibiotic was administered prior to the diagnosis of meningitis in six patients.

\section{Outcome}

Two patients with infarction on CT died. Ten of the surviving patients had neurologically un-

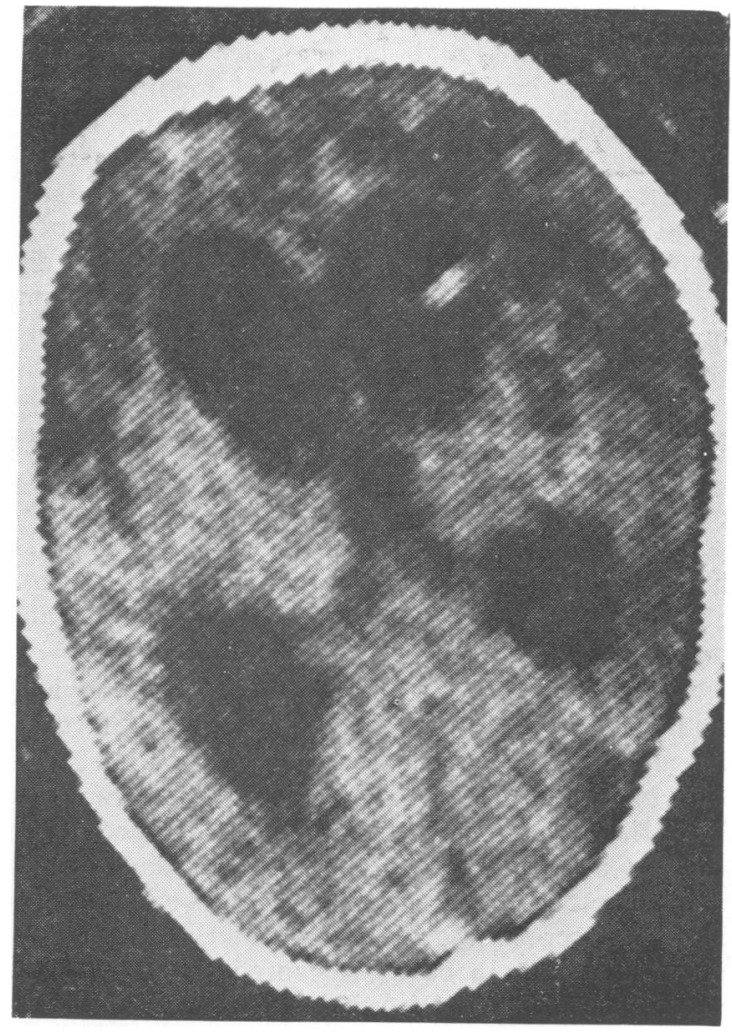

Fig 4 CT performed 5 weeks after pneumococcal meningitis and 2 weeks after placement of $a$ ventriculoperitoneal shunt with the ventricular end in the right frontal horn. Age seven months. Multiple frontal and right posterior low density areas.

Ventricular enlargement was also present preoperatively and did not change following shunt. Fontanelle never full.

favourable outcome. Sequelae in this group consisted of mental retardation, spastic tetraparesis, cortical blindness, and seizures.

\section{Discussion}

The presence of vascular lesions in childhood bacterial meningitis has been noted by pathologists for many years. Wertham,${ }^{15}$ in 1931 , when reviewing necropsy data from children and adults, commented upon the frequency of cerebral lesions in purulent meningitis and attributed these lesions to circulatory factors. Adams, Kubik, and Bonner ${ }^{4}$ performed a necropsy study of children with meningitis and found pathological changes in brain parenchyma identical to those of recent infarction. These authors also noted neutrophils 
extending into the perivascular spaces of the cortex. Neutrophils and lymphocytes were found beneath the initima of small and medium sized subarachnoid arteries. Focal necrosis and mural thrombi occurred in veins. Smith and Landing, ${ }^{5}$ in another review of the pathology of childhood bacterial meningitis, recognised the relative frequency of phlebitis in the subarachnoid exudate. They felt that phlebitis may be an important mechanism in the neurological deficit. Arterial thromobosis was rare in their study.

Cerebral angiography has revealed vascular lesions in children with bacterial meningitis. ${ }^{91016}$ Abnormalities occurred in cerebrovascular dynamics as well as arteritis, thrombosis, thrombophlebitis, and vascular narrowing. These reports have provided further evidence of the severe abnormalities in cerebral blood flow which accompany bacterial meningitis in childhood.

Our findings on CT confirm these pathological and radiographic examinations. Focal cortical areas of decreased density without major mass effect were demonstrated by CT in children with bacterial meningitis. The areas of decreased density often occurred in a vascular distribution and were felt to be compatible with areas of infarction (cerebrovascular accidents) secondary to a vasculitis produced by the infectious process in vessels entering and leaving the brain. ${ }^{17} \mathrm{~A}$ brain biopsy in one patient was taken from an area of CT low density. The biopsy revealed the pathological findings of infarction thus helping to establish the association of cortical low density lesions on CT and infarction. However, focal areas of cortical necrosis secondary to some other factor, although unlikely, could not be completely excluded in other patients. Hemiparesis was relatively uncommon, perhaps reflecting the bilateral occurrence of the lesions.

CT evidence of infarction occurred in several children who had the diagnosis of meningitis established within two days of onset of symptoms of a febrile illness, and had relatively rapid institution of appropriate antibiotic therapy. Thus, delay in institution of appropriate antimicrobial therapy may not be causally related to infarction in all cases. Low CSF glucose concentration occurred in seven cases, but its significance in infarction is uncertain. Eleven of the children with CT evidence of infarction also had CT evidence of ventricular enlargement. We were unable to determine conclusively whether this was related to increased intracranial pressure or to a relatively acute form of brain atrophy. Perhaps both mechanisms played a part. Follow-up CT, at least one month after the initial scan, was performed in five of the patients with ventricular enlargement. The enlargement persisted and, in three patients, was associated with a head circumference which was progressively falling below the normal curve. This provided further evidence for the clinical suspicion that pressure hydrocephalus was not occurring.

Our studies suggest that factors exist in childhood bacterial meningitis which are associated with the development of cerebral infarction. These factors include age less than one year, infection with $S$ pneumoniae, severe hypoglycorrachia, early inappropriate antibiotic therapy, and male sex (table). Seizures occurred in all of our patients; it was our clinical impression that the seizures heralded the occurrence of infarction, but did not cause infarction. Even with early diagnosis and prompt institution of appropriate antibiotic therapy, CT evidence of cerebral infarction occurred.

Table Factors associated with cerebral infarction $(N=13)$

Age less than one year

Seizures

Male sex

Unfavourable outcome

Severe hypoglycorrhacia $-<0.6 \mathrm{mmol} / 1$

Cerebral infarction in survivors of childhood bacterial meningitis appears to be a serious, relatively common complication that may have been overlooked by clinicians prior to the availability of CT. Bacterial meningitis may produce a vasculitis of cerebral vessels with consequent thrombosis and ischaemic infarct. Meningitis is a disease process which may exert a profound secondary effect on the brain parenchyma.

\section{References}

1 Dodge PR, Swartz MN. Bacterial meningitisa review of selected aspects. New Eng J Med 1965; 272:954-60.

2 Sell SHW, Merrill RE, Doyne EO, Zimsky EP. Long-term sequelae of Hemophilus influenzae meningitis. Pediatrics 1972; 49:206-17.

3 Lindberg J, Rosenhall U, Nylen O, Ringner A. Long-term outcome of Hemophilus influenzae meningitis related to antibiotic treatment. Pediatrics 1977; 60:1-6.

4 Adams RD, Kubik CS, Bonner FJ. The clinical and pathological aspects of influenzal meningitis. Arch Pediatr 1948; 65:354-80, 408-59.

5 Smith JF, Landing BH. Mechanism of brain damage in $H$. influenzae meningitis. J Neuropathol Exp Neurol 1960; 19:248-65. 
6 Dodge PR, Swartz MN. Bacterial meningitisa review of selected aspects. New Eng J Med 1965; 272:1003-10.

7 Leeds NE, Goldberg HI. Angiographic manifestations in cerebral inflammatory disease. Radiology 1971; 98:595-604.

8 Headings DL, Glasgow LA. Occlusion of the internal carotid artery complicating Hemophilus influenzae meningitis. Am J Dis Child 1977; 131: 854-6.

9 Gado M, Axley J, Appleton DB, Prensky AL. Angiography in the acute and post-treatment phases of Hemophilus influenzae meningitis. Radiology 1974; 110:439-44.

10 Raimondi AJ, Di Rocco C. Cerebral angiography in meningocerebral inflammatory disease in infancy and childhood: a study of thirty-five cases. Neurosurgery 1978; 3:37-44.

11 Bachman DS, Hodges FJ, Freeman JM. Com- puterized axial tomography in neurologic disorders of children. Pediatrics 1977; 59:352-63.

12 Cockrill HH, Dreisbach J, Lowe B, Yamauchi T. Computed tomography in leptomeningeal infections. Am J Roentgenol 1978; 130:511-5.

13 Weisberg LA. Cerebral computerized tomography in intracranial inflammatory disorders. Arch Neurol 1980; 37:137-42.

14 Stovring J, Snyder RD. Computed tomography in children bacterial meningitis. J Pediatr 1980; 96:820-3.

15 Wertham $F$. The cerebral lesions in purulent meningitis. Arch Neurol Psychi 1980; 26:549-82.

16 Lyons EL, Leeds NE. The angiographic demonstration of arterial vascular disease in purulent meningitis. Radiology 1967; 88:935-8.

17 Kinkel WR, Jacobs L. Computerized axial transverse tomography in cerebrovascular disease. Neurology (Minneap) 1976; 26:924-30. 